\section{Forecasting System Monitoring under Non-normal Input Noise Distributions}

\section{Hoda Sabeti, Omar Al-Shebeeb and Majid Jaridi*}

Department of Industrial Engineering, West Virginia University, Morgantown, West Virginia, USA

*Corresponding author: Jaridi M, Department of Industrial Engineering, West Virginia University, Morgantown, West Virginia, USA, Tel: +(304) 293-4099; E-mail: majid.jaridi@mail.wvu.edu

Received date: June 17, 2016, Accepted date: June 28, 2016, Published date: June 30, 2016

Copyright: (c) 2016 Sabeti $\mathrm{H}$, et al. This is an open-access article distributed under the terms of the Creative Commons Attribution License, which permits unrestricted use, distribution, and reproduction in any medium, provided the original author and source are credited.

\begin{abstract}
In quantitative forecasting models and tracking signal methods, input noise is often assumed to be normally and independently distributed. The goal of this research was to study the distribution of tracking signal and build new monitoring schemes for when the input noise distribution is not necessarily normal. A demand process in the Wilson inventory model was simulated using several input noise distributions. The effectiveness of a proposed tracking signal model was evaluated and compared to existing methods using an inventory cost model. It was found that it is not realistic to assume a normal distribution for the tracking signal even when the noise is normal. Because of the dependency of tracking signal elements, and since there is no specific distribution for it, we used simulation to estimate the best value for the standard deviation and suggest $\pm 3 \widehat{\sigma}_{T S}$ as the control limits. We compared this value with those suggested by other papers, and showed that the proposed limits work better when the process is under control and also when there are different amounts of shifts in mean demand. We also studied different values for the tracking signal smoothing parameter and analyzed the inventory costs for each.
\end{abstract}

Keywords: Quantitative forecasting; Tracking signal; Inventory control

\section{Introduction}

The aim of a forecast monitoring system is to monitor how well a system is functioning, to detect "out of control" signals expeditiously, and to provide an unbiased estimate of the forecast error. This monitoring can be achieved efficiently with the use of a "tracking signal." Tracking signal methods have been used as alarm mechanisms to ensure that forecasting systems remain in control, and to detect any abnormalities in a timely manner in order to provide information to decision makers. This allows for appropriate corrective action to be taken so that the forecasting system can be brought back to an "under control" status.

Time series forecasting systems as applied to inventory systems have been extensively studied using many different types of tracking signals. Brown [1] was the first to suggest the use of tracking signals, and subsequent researchers such as Trigg continued and expanded upon Brown's tracking signal method [2]. Some of these researchers also suggested new methods of using tracking signals. For example, Deng et al. used fuzzy-neural to suggest a new tracking signal [3].

To be confident that the tracking signal provides reasonable assurance against missing out of control signals (type II error), and balancing this with the probabilty of false alarms (type I error), it is important to calculate control limits carefully and include the costs of committing type I and type II errors in such calculations.

Many researchers calculate tracking signal control limits by assuming that the input noise is normally distributed. The main goal in this paper is to answer the question "What is the effect on the control limits if the input noise is not normally distributed, but rather follows some other distribution such as Beta or Gamma?" The second question that follows the first is "How will the performance of the tracking signal be affected with these new control limits?"

\section{Literature Review}

Tracking signal methods have been used to check the bias of forecasting methods or the change of a demand process over time. The first form of a Tracking Signal (TS) was developed by Brown [1]. Brown proposed the Cumulative Sum test (CUSUM) as a tracking signal to control inventory forecast. Briefly, the CUSUM TS is the ratio of the cumulative errors to the Mean Absolute Deviation (MAD). This method contains disadvantages, such as when the tracking signal goes out of control limits because the forecasting system has gone out of control, in which case the tracking signal may not return to its under control state even though the forecasting system has returned back to control. Additionally, even with an unbiased and accurate forecasting system, the tracking signal may go outside the control limits in the event that the MAD approaches zero and the cumulative error continues to grow.

Trigg [2] updated Brown's [1] tracking signal by developing a smoothed error tracking signal. This was accomplished by dividing the smoothed error $\left(E_{t}\right)$ by the MAD [2]. Then Trigg et al. [4] developed adaptive exponential smoothing to improve the tracking signal performance. Trigg et al. [4] proposed a method of varying the response rate of the system according to the value of the tracking signal. Trigg et al. [4] suggested the ratio of $\left(E_{t}\right)$ divided by smoothed absolute error $\left(\mathrm{MAD}_{\mathrm{t}}\right)$ to be used as the value of the smoothing constant at for the next period of forecasting. Brown later showed how to calculate the best smoothing constant [5]. Alstrøm et al. [6] evaluated the quality of the tracking signal by how well it performed in an inventory control system. They depended on the ability of the tracking signal to detect changes in the level, trend and seasons on five different demand processes. 
Ravi [7] made the assumption that the demand values in different periods are independent and identically distributed (IID), as well as normal. He suggested a simple modification to the tracking signal to fix the flaw with the common assumption that the demand values in different periods are IID. This modified unsmoothed tracking signal computed at the end of period it was named "AVGMADTSt," and they analyzed the AVGMADTSt for a range of $\mathrm{N}$ values, where $\mathrm{N}$ is the number of periods in months. Deng et al. developed a Neuro-fuzzy model to propose a new smoothed error tracking signal test [3].

CUSUM has been used in many applications, including medicine and education. Biau et al. [8] used a CUSUM control chart to monitor surgeon performance on knee replacement surgeries. Mele et al. [9] used two time series models, the moving average model (MAM) and the tracking signal model (TSM), for detecting oestrus and udder health in dairy cows. Mele et al. [9] confirmed that the use of the TSM was advantageous because it reduced the number of false positives in healthy cows.

A tracking signal works as an alarm for any bias in the forecasting system if the forecasting process is in control, and in another range if it is out of control. Some researchers use three standard deviations [10] for the control limits, and assume that the tracking signal is normally distributed. Trigg [2] showed that the standard deviation of smoothed error can be estimated from the relationship $\sigma=1.2 \mathrm{MAD}$, where $\sigma$ is the standard deviation and the two sigma control limits for the tracking signal are $\pm 2.4 \sqrt{\left(\frac{\alpha}{2-\alpha}\right)}$, where $\alpha$ is the smoothing constant. McClain [11] developed a new method to automatically monitor an exponentially smoothed forecast. Ristroph [12] stated that "Approximate confidence limits can be derived by first considering the ratio of the smoothed error to its standard deviation".

Some researchers have attempted to evaluate the performance of various tracking signals. To the best of our knowledge, all prior studies on tracking signals consider the input noise to be normally distributed. Brown showed that the forecasting errors from exponential smoothing are normally distributed if the input noise is also normally distributed [1]. Moreover, many researchers [2,4,9-15] have considered the forecasting errors from exponential smoothing to be normally distributed since the input noise is normally distributed.

Batty [13] assumed that the distribution of the error and the smoothed error was normal, and developed new equations to calculate the MAD and the control limit of the tracking signal. McKenzie [10] derived some statistical properties of tracking signals. McKenzie [10] states that a tracking signal is "the ratio of two correlated random variable each of which is the most recent element of a stochastic process, it is extremely difficult to derive the distribution of the signal.' McKenzie [10] suggests making some simplifying assumptions to approximate the mean and variance, but these assumptions lead to considering a normal distribution for the error. Alstrøm et al. [6] ignored the real distribution of tracking signals and overestimated its standard deviation. For Brown's[1] TSM, the over-estimated standard deviation is $\sigma_{T S}=0.88 \sqrt{\frac{2-\alpha}{1-(1-\alpha)^{2}}}$, where Brown [1] used $\pm 3 \sigma \mathrm{TS}$ as control limits for the tracking signal, where $\alpha$ is the smoothing constant. For Trigg's [4] tracking signal, the standard deviation is $\sigma_{T S}=0.88 \sqrt{\alpha}$. McKenzie [10] used a different smoothing constant in the forecasting system and showed that the use of an appropriate smoothing constant greatly improves the performance of the tracking signal compared to the CUSUM scheme.
None of the above researchers consider non-normal distributions for the input noise. In this research we consider normal, Beta, and Gamma distributions for the input noise, and we investigate the distributions of the tracking signals. Additionally, we consider an inventory system similar to the one used by Alstrøm et al. [6]. Matlab R2013a was used to simulate the demand process and the inventory system. Two different demand processes were considered. In the first process, the demand is level with constant variance and is subject to random changes. In the second process, the demand has a trend and its mean changes during the study time period with the same pattern as the first process.

As mentioned before, all past studies have considered the input noise to be normally distributed. In this paper, we consider three different distributions for the input noise and study their effects on the tracking signal distribution and control limits. In the level demand process, three different random data distributions were generated. First, authors assumed that the input noise is normally distributed. The second distribution is a Beta distribution with $\alpha=\beta=a$, where $a>1$, as parameters. Finally, the third distribution is a Gamma distribution.

The tracking signal suggested by Trigg [4] was used here. In the first step authors attempted to fit some distributions to the tracking signal value and build control limits based on them. Then, authors ran the inventory model using the proposed control limits and those suggested by Trigg [4] and Alstrøm et al. [6]. The effectiveness of the TSMs was evaluated under in-control and out-of-control demand processes.

This paper is organized as follows. The methodology is presented in Section 3. Subsection 3.1 describes the demand process, and the input noise distributions are discussed in 3.2. Subsection 3.3 and 3.4 illustrate the forecasting models and tracking signal methods, respectively. Subsection 3.5 introduces the inventory model and the costs related to inventory forecasting. Section 4 presents the numerical results of the simulated data and the distribution of tracking signal. A discussion of the control limits for each follows. An approach to the determination of control limits is proposed, and a sensitivity analysis related to the smoothing parameter is presented. The conclusions and recommendations for future research are provided in Section 5.

\section{Methodology}

The goal of this research was to study the distribution of a tracking signal and build new monitoring schemes when the input noise distribution is not necessarily normal. The input noise distributions considered here are Normal $(0, \sigma 2)$, Beta, and Gamma. Authors simulated these conditions by generating a level demand process (explained in Subsection 3.1) using several input noise distributions (described in Subsection 3.2). After generating random demand data using the above input noise distributions, we used the simple exponential smoothing method to forecast the demand (Subsection 3.3). We then used Trigg's [2] TSM and Trigg et al. [4] adaptive smoothing parameter method to monitor future forecast errors and calculate the tracking signal for each period (Subsection 3.4). Next, authors experimented with fitting several distributions to the tracking signal and found new limits for controlling the forecast error using those tracking signals (Subsection 3.5). Finally, authors used an inventory cost model to evaluate the performance of the proposed tracking signal limits and compared it to the other limits suggested by Trigg et al.s [4] and Alstrøm et al. [6] under in control and out of control demand processes (Subsection 3.6). 
Page 3 of 7

\section{Demand process}

To study the tracking signal distribution characteristics and evaluate its performance while the underlying process is changing, the following level demand process is considered here:

$$
x_{t}=\mu+\varepsilon_{t}(1)
$$

Where $x_{\mathrm{t}}$ is the demand at time $\mathrm{t}, \mu$ is the mean demand, and $\mathcal{E}_{\mathrm{t}}$ is the random error component. In this process, there may be random shifts in mean demand over time. The distribution of the random error component in the demand process will be discussed later in Subsection 3.2. The mean changes between various time periods, but remains fixed within each period. Figure 1 shows an example of a level demand process.

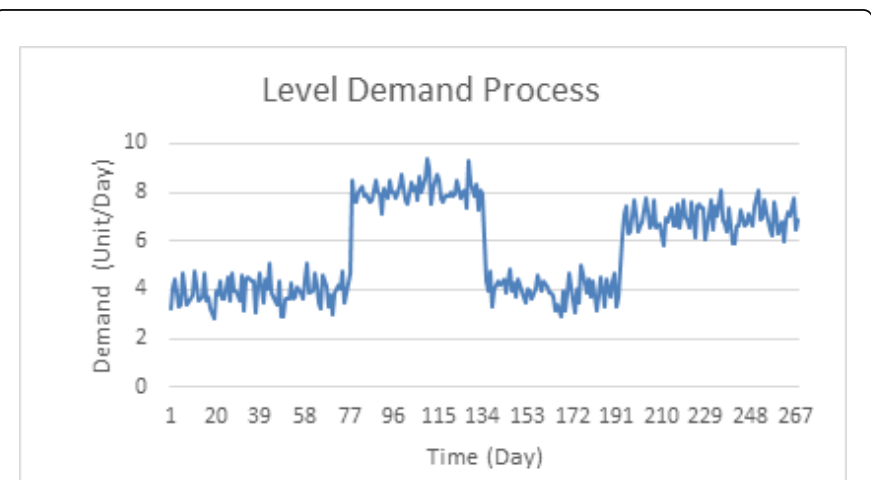

Figure 1: An example of a level demand process with shifts in mean demand.

\section{Input Noise Distribution}

To study the effect of different input noise distributions on the tracking signal distribution, we generated demand processes with three different input noise distributions as follows:

\section{Normal distribution}

In most forecasting literature, the input noise distribution is considered to have a normal distribution. In this paper, we too consider the Normal $\left(0, \sigma_{\varepsilon}^{2}\right)$, distribution as one of the input noise distributions to compare the results with those obtained using two other distributions shown below.

\section{Beta distribution}

Since the Beta distribution is a general form of many different distributions, here we used a Beta distribution with parameters $\alpha=\beta>1$.

\section{Gamma distribution}

The Gamma distribution is also a general form for several other distributions. Therefore, we considered a Gamma distribution with parameters $\alpha$ and $\beta$ for the input noise.

\section{Forecasting Model}

In the level demand process, simple exponential smoothing was used as an effective forecasting method. The simple exponential smoothing equation can be written as:

$$
\begin{aligned}
& s_{0}=x_{0}(2) \\
& s_{t}=\alpha x_{t-1}+(1-\alpha) s_{t-1}
\end{aligned}
$$

Where:

$x_{\mathrm{O}}$ is the actual demand in the first period,

$x_{\mathrm{t}-1}$ is the actual demand in period $(\mathrm{t}-1)$,

$s_{\mathrm{t}}$ is the demand forecast for period $\mathrm{t}$ (i.e., the value of the smoothed statistic at the end of period $t)$, and

$\alpha$ is the smoothing constant, $0 a 1$.

\section{Tracking Signal Method}

As mentioned before, different tracking signal methods have been developed to monitor the forecasting process. In this paper, we consider the tracking signal introduced by Trigg [2] and the adaptive smoothing parameter suggested by Trigg et al. [4]. Trigg's tracking signal method can be expressed as follows [2]:

\section{Tracking Signal=Smoothed Error/Smoothed Absolute} Error $=S E / S A E(4)$

$$
\begin{gathered}
S E_{t}=(1-\beta) S E_{t-1}+\beta^{*} E r_{t} \\
S A E_{t}=(1-\beta) S A E_{t-1}+\beta^{*}\left|E r_{t}\right|(6)
\end{gathered}
$$

Where $\mathrm{SE}_{t}$ is the smoothed error at time $t, \mathrm{SAE}_{t}$ is the smoothed absolute error at time $t, \mathrm{Er}_{\mathrm{t}}$ is the error at time $\mathrm{t}$, and $\beta$ is the tracking signal parameter. Trigg stated that if the process is in control then the tracking signal will occur around zero within the range \pm 1 [4]. A large tracking signal value indicates a bias in the forecast.

For the tracking signal parameter, $\beta$, we initially used the forecasting parameter $\alpha$. However, based on Gardner et al. [16] and Batty [13], the tracking signal has a better performance if

\section{$\beta<\alpha(7)$}

In order to study the best performance of a tracking signal, we also performed a sensitivity analysis on the value of as a proportion of $\alpha$, and the results are presented in Section 3.

Trigg et al. [4] suggested the use of the following equation when the process goes out of control to adapt the response rate:

$$
\alpha=\text { modulus of tracking signal (8) }
$$

They suggested using the tracking signal value as the forecasting parameter to adjust the next period's forecasting. In this paper, we used this method when there was a shift in the mean demand and the tracking signal model showed the process was out of control.

\section{Tracking Signal Distribution and Control Limits}

The methods explained in Subsection 3.4 were used to calculate the tracking signal for each period when the process was under control and there were no shifts in the mean demand. In the next step, we analyzed the tracking signal distribution. We fit several distributions (Normal, Beta, Gamma, Weibull, and Exponential) to either the 
Page 4 of 7

tracking signal data or their absolute value. The Chi-square test was applied to test the goodness of fit of these distributions. As shown in Section 4, Chi-square test rejected the goodness of fit for these distributions.

Since we could not fit a specific distribution to the TS values, we tried to estimate its variance and build the control limits based on this estimated variance. Based on Trigg et al. [4], Brown [1], and Alstrøm et al. [6], there is a relationship between the TS constant and its variance.

Trigg et al. [4] provided the following approximate value for the tracking signal's standard deviation:

$$
\widehat{\sigma}_{\mathrm{TS}}=\sqrt{\frac{1.5^{*} \beta}{2-\beta}}(9)
$$

Alstrøm et al. [6] provided the following somewhat overestimated value for the tracking signal's standard deviation:

$$
\widehat{\sigma}_{\mathrm{TS}}=0.88 * \sqrt{\beta}
$$

\section{where $\beta$ is the smoothing parameter for tracking signal.}

We tried different relationships in our simulation models and used regression to find the best relationship between the variance of the tracking signal and its corresponding smoothing parameter. Based on our simulation models, the standard deviation of the tracking signal was estimated as:

$$
\widehat{\sigma}_{\mathrm{TS}}=.85^{*} \beta+.125(11)
$$

In the next step, the estimated standard deviation was used to set control limits for the tracking signal. We then established a monitoring model for the future demand forecast. We used an inventory model (Subsection 3.6) to compare this suggested model with Trigg et al. [4] and Alstrøm et al. [6]. Next, we generated some shifts in the process level and evaluated the performance of the tracking signal by calculating the inventory costs.

\section{Inventory Models}

The ability of the tracking signal method to detect shifts in the levels and trends is measured by the changes in inventory costs. Here we used the Wilson Formula and a (B, Q) system, which consists of an order quantity and a reordering point. The order quantity is calculated using the Economic Order Quantity (EOQ) formula, and is updated each month since the forecasted demand changes each month.

$$
Q=\sqrt{\frac{2 A D}{H}}
$$

Where $\mathrm{A}$ is the ordering cost per order, $\mathrm{D}$ is the average forecasted demand for the next period, and $\mathrm{H}$ is the holding cost per unit of product.

The order point should be large enough to cover stock-outs during the lead-time. This can be determined using the following inventory theory equation:

\section{B=Safety Stock+Mean Demand During Lead - Time (13)}

Safety stock can be calculated using a safety factor and the variance of demand during the lead-time. Here we do not assume a normal distribution for demand during the lead-time. Instead, we use the forecasted lead-time demand and update the order point each month based on it.

Finally, the following equation is used to calculate the total cost:
Total Cost=Holding Cost + Ordering Cost + Stock Out Cost (14)

\section{Numerical Results}

\section{Tracking signal distribution and control limits}

In this study, we consider three cases of different input noise distributions. However, we generated the same level demand process for each case. The length of the process ranged between 40 and 400 periods, and the first 20 periods were used to find the best smoothing forecast constant, $\alpha$, which minimizes the mean squared error (MSE).

We calculated the tracking signal for these periods and attempted to fit a distribution to them. Figure 2 shows the results of fitting distributions for 5,000 runs with normal input noise.

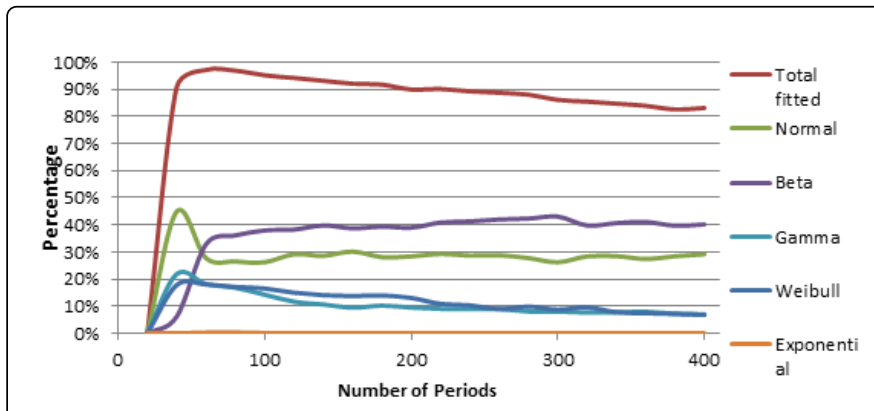

Figure 2: The percentage of each fitted distributions and the total number of fitted distributions among 5,000 runs for normal $(0,0.5)$ input noise.

Figure 2 shows the percentages of different fitted distributions and also the percentage of the total number of fitted distributions when the input noise is normal. For example, when the process length is 100 periods, among 5,000 runs, $38 \%$ of the time the Chi-squared test does not reject the goodness of fit of a Beta distribution. It does not reject the goodness of fit for Normal, Weibull, and Gamma distributions $26 \%, 17 \%$, and $15 \%$ of the time, respectively. In general, when the input noise is normally distributed and the process length is 100 months, 95\% of the time the Chi-squared test does not reject the goodness of fit for some distribution.

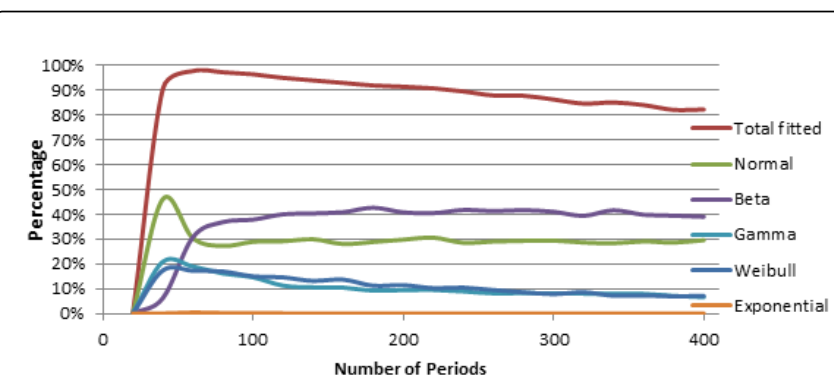

Figure 3: The percentage of each fitted distribution and the total number of fitted distributions among 5,000 runs for Beta $(2,2)$ input noise.

Figure 2 also shows that the total number of fitted distributions decreases as the number of periods increases. Normal distribution is at best only $45 \%$ of the total runs, and this number decreases as the 
number of period's increases. In other words, assuming a normal distribution for the tracking signal is not a realistic assumption even when the input noise is normal.

Figures 3 and 4 show the same results for Beta $(2,2)$ and Gamma $(8$, 0.25 ) input noise, respectively. These figures indicate that when the input noise is not normal, the result is similar. These figures show that it is hard to fit a particular distribution to the tracking signal, especially as the number of runs increases.

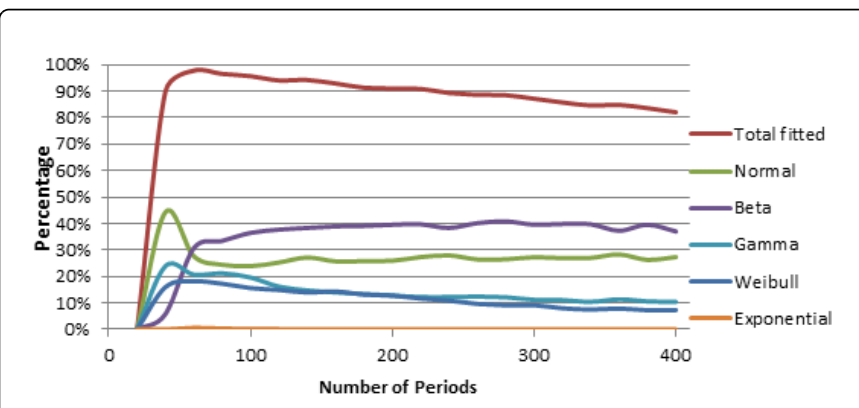

Figure 4: The percentage of each fitted distribution and the total number of fitted distributions among 5,000 runs for Gamma (8, $0.25)$ input noise.

\section{In-control process (no shift in the demand process)}

We then tried to find a good estimate of the tracking signal variance to build control limits around. As explained in Subsection 3.5, the following relationship was used to find the variance of tracking signal:

$\widehat{\sigma}_{\mathrm{TS}}=.85^{*} \beta+.125$ (13) The control limits are defined as $\pm 3 \widehat{\sigma}_{T S}$.

To test the capabilities of this monitoring method, we ran the simulated inventory model 5,000 times for each of the following cases and compared the cost of different methods.

Case 1: not using TS and only use forecasted demand.

Case 2: using control limits to monitor the process [4].

Case 3: using control limits to monitor the process [6].

Case 4: using suggested control limits.

Case 5: using the real demand as the forecast to minimize cost for the cost index.

The parameters for the inventory simulation were as follows: Holding Cost $=\$ 25$ per unit, Backorder Cost $=\$ 35$ per unit, and Ordering Cost $=\$ 100$ per order, and the lead time is 2 periods Figure 5.

In this case, there is no shift in mean demand and the process is under control. Figures 5-7 present the mean inventory costs in 5,000 runs for each method with different input noise distributions. To have a better comparison, we considered a case where real demand was used as the forecasted one and the forecast was $100 \%$ accurate. This minimum cost was used to find an index for the other methods' costs.

Figures 5-7 show that there is not much of a difference between the TSMs when the process is in control, as the range of the index is very narrow. For the normal noise, Figure 5 shows that using the forecast method without the tracking signal has a cost index of 103.37 and other methods are grouped around this value. The Trigg et al. [4] and
Alstrøm et al. [6] methods overlap. The proposed method moves closer to the line and shows slightly lower costs, but the difference is negligible. Figures 6 and 7 show similar results. The difference between methods and not using a tracking signal is small. Since the process is in control and there is no shift in mean demand, this result was expected.

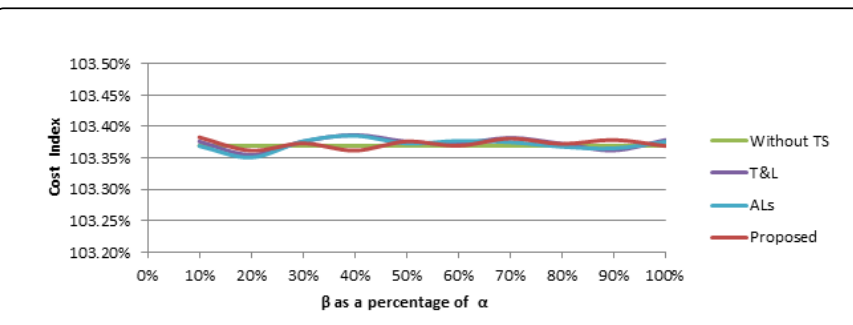

Figure 5: The cost index of different tracking signals when the noise is Normal $(0,0.5)$ and the process is in control.

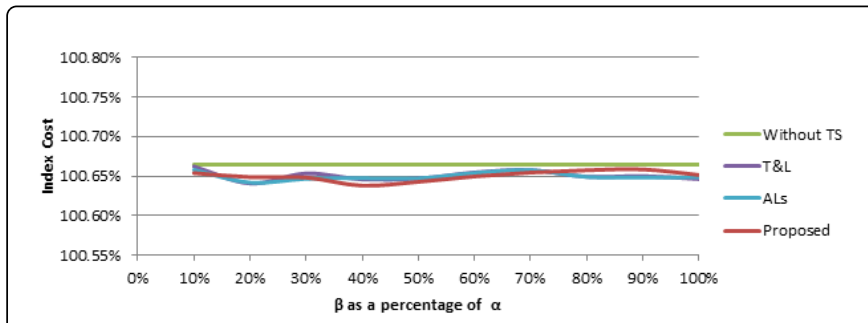

Figure 6: The cost index of different tracking signals when the noise is Beta $(2,2)$ and the process is in control.

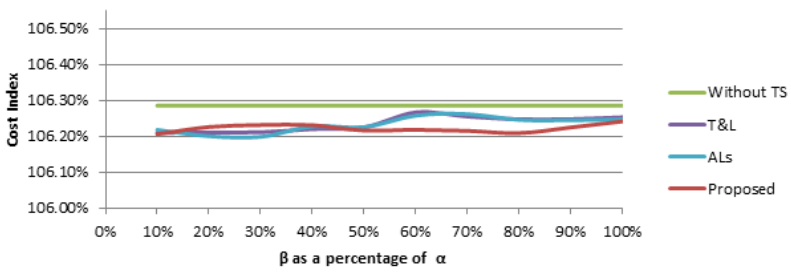

Figure 7: The cost index of different tracking signals when the noise is Gamma $(8,0.25)$ and the process is in control.

Based on Alstrøm et al. [6] and Batty [13] the tracking signal has a better performance if $\beta<\alpha$. Here we set $\beta$ as a percentage of $\alpha$, and the horizontal axis shows this percentage. Figure 6 indicates that when the noise is normal, the proposed method works better than the others when $0.30 \alpha<\beta<0.65 \alpha$. When the input noise is Beta or Gamma distributed, the proposed method shows lower costs when $0.40 \alpha<\beta<0.70 \alpha$. However, since the process is in control, different values of $\beta$ do not affect the cost significantly. In the next subsection, we generate some shifts to the mean demand and study the effect of the $\beta$ value.

\section{Out-of-control process (shifts in the demand process)}

Until now, the process was assumed to be under control. Now we need to check the tracking signal's ability to detect changes in the underlying process. To do this, we shifted the mean for several periods 
Page 6 of 7

and then reverted it back to its original value. Two different shifts were considered here. The first shift was twice the mean demand, and the second shift was half of the mean demand. Then all the steps followed in the previous subsection were repeated, and the results are shown in Figures 8-10.

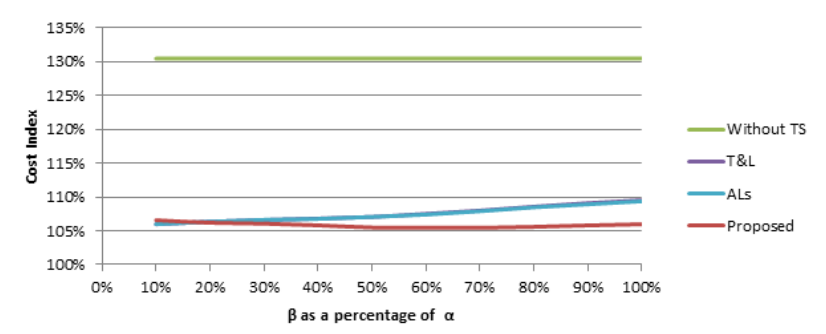

Figure 8: The cost index of different tracking signals when the noise is Normal $(0,0.5)$ and mean demand shifts.

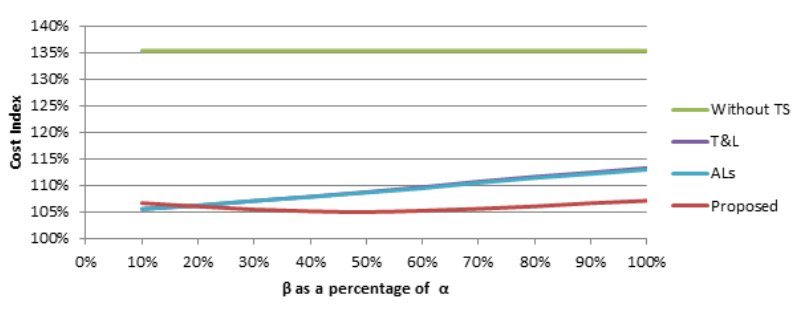

Figure 9: The cost index of different tracking signals when the noise is Beta $(2,2)$ and there are shifts in mean demand.

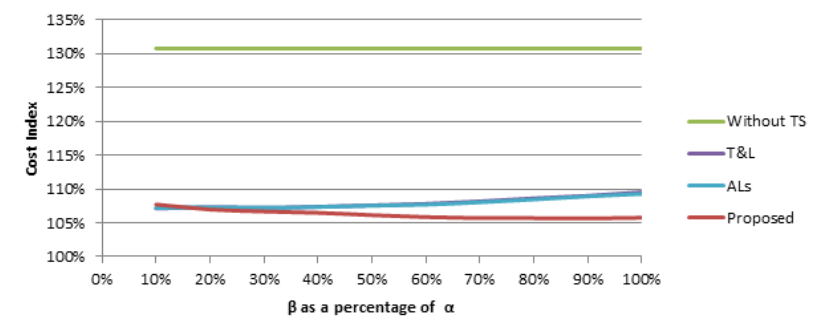

Figure 10: The cost index of different tracking signals when the noise is Gamma $(8,25)$ and there are shifts in mean demand.

Figure 8 shows the case where the underlying input noise has a normal distribution. In this case, using the forecasting method without a tracking signal can raise the cost index to $130 \%$, while using the proposed control limits can reduce costs to just over $105 \%$. The lower costs are in the range of $0.55 \alpha<\beta<0.75 \alpha$ The Trigg et al. [4] and Alstrøm et al. (1996) control limits are very close. As the value of $\beta$ as a percentage of $\alpha$ increases, the difference in cost for different methods becomes more prominent.

Figure 9 indicates similar results for the Beta distribution. Using tracking signal methods can reduce the costs by up to $30 \%$. If the tracking signal is constant and $0.35 a<\beta<0.65 a$, the cost index can be reduced to $105 \%$. Here again, the Trigg et al. [4] and Alstrøm et al. [6] models produce similar results and the proposed control limits result in lower costs.

For the Gamma input noise distribution, Figure 10 shows up to $25 \%$ decrease in costs when using the proposed control limits. The best savings occur in the range of $0.60 \alpha<\beta<0.95 \alpha$. In general, $0.60 a<\beta<0.70 a$ tends to decrease overall inventory costs.

\section{Conclusion}

In this paper we considered different distributions for the input noise and studied the distribution of a tracking signal. We showed that assuming a normal distribution for the tracking signal is not realistic even when the noise is normal. Because of the dependency of tracking signal elements, and since there is no specific distribution for it, we used simulation to estimate the best value for the standard deviation and suggested $\pm 3 \widehat{\sigma}_{T S}$ as the control limits. We compared this value with those suggested by other results reported in the literature. Results in Section 3 show that the proposed limits work better when the process is under control and also when there are different sized shifts in mean demand.

We also studied different values for the tracking signal smoothing parameter and analyzed the resulting inventory costs. For the proposed method, we found that using a value between $60-70 \%$ of the forecasting parameter produces the minimum cost. Future researchers may wish to consider other demand processes, such as the trend line demand process. Our work could also be expanded upon to consider seasonality in the demand process.

\section{References}

1. Brown RG (1959) Statistical forecasting for inventory control, McGrawHill, USA. pp: 232.

2. Trigg D (1964) Monitoring a forecasting system. Operational Research Society $15: 271-274$.

3. Deng Y, Jaraiedi M, Iskander W (2004) Tracking signal test to monitor an intelligent time series forecasting model. Intelligent Manufacturing, SpieInt Soc Optical Engineering 149.

4. Trigg D, Leach A (1967) Exponential smoothing with an adaptive response rate. Journal of the Operational Research Society 18: 53-59.

5. Brown RG (2004) Smoothing, forecasting and prediction of discrete time series. Dover Publication Inc. Mineola, Newyork.

6. Alstrøm P, Madsen P (1996) Tracking signals in inventory control systems A simulation study. International Journal of Production Economics 45: 293-302.

7. Ravi PS (2013) An analysis of a widely used version of the CUSUM tracking signal. Journal of the Operational Research Society 65: 1189-1192.

8. Biau D, Meziane M, Bhumbra R, Dumaine V, Babinet A, et al. (2011) Monitoring the quality of total hip replacement in a tertiary care department using a cumulative summation statistical method (CUSUM). Journal of Bone and Joint Surgery 93: 1183-1188.

9. Mele M, Secchiari P, Serra A, Ferruzzi G, Paoletti F, et al. (2001) Application of the 'tracking signal'method to the monitoring of udder health and oestrus in dairy cows. Livestock Production Science 72: 279-284.

10. McKenzie E (1978) The monitoring of exponentially weighted forecasts. Journal of the Operational Research Society 29: 449-458.

11. McClain JO (1988) Dominant tracking signals. International Journal of Forecasting 4: 563-572.

12. Ristroph JH (1990) Monte Carlo modeling of the tracking signal for forecast errors in computer integrated manufacture. Computers \& Industrial Engineering 19: 67-71. 
Citation: Sabeti H, Al-Shebeeb O, Jaridi M (2016) Forecasting System Monitoring under Non-normal Input Noise Distributions. Ind Eng Manage 5: 194. doi:10.4172/2169-0316.1000194

Page 7 of 7

13. Batty M (1969) Monitoring an exponential smoothing forecasting system 20: 319-325.

14. Snyder RD, Koehler AB (2009) Incorporating a tracking signal into a state space model. International Journal of Forecasting 25: 526-530.

15. Dobrota M, Vujošević M (2015) Forecasting and Inventory Performance in Direct-Store Delivery Supply Chain: Case of Retailer in Serbia. International Journal for Traffic and Transport Engineering 5.
16. Gardner ES (1985) CUSUM vs. smoothed-error forecast monitoring schemes: Some simulation results. Journal of the Operational Research Society $36: 43-47$. 